\title{
AN ACCURATE ANALYTICAL MODEL FOR NONEQUILIBRIUM DRIFT-VELOCITY AND CHORD-MOBILITY OF In 0.53 $_{0.47} \mathrm{Ga}_{0.4}$
}

\author{
E. Moreno ${ }^{\mathrm{a}}$ and L. Varani ${ }^{\mathrm{b}}$ \\ a Astro-Photonics Lab, Department of Electrical Engineering at the Beauchef Campus, University of Chile, \\ Av. Tupper 2007, 8370471 Santiago de Chile, Chile \\ ${ }^{\mathrm{b}}$ Institute of Electronics and Systems, University of Montpellier, Bâtiment 5, 860 rue de St Priest, 34090 Montpellier, France \\ Email: enrique@moreno.ws; luca.varani@umontpellier.fr
}

Received 27 October 2017; revised 17 November 2017; accepted 21 June 2018

\begin{abstract}
Mobility models are an essential tool for an accurate description of the charge carrier dynamics in semiconductor materials and devices. By means of a simulator based on the Monte Carlo method which has been properly validated, a set of velocity and chord-mobility data was generated for electrons and holes in $\mathrm{In}_{0.53} \mathrm{Ga}_{0.47}$ As bulk material as a function of electric field and for different concentrations of donors and acceptors. This set has been used to build an accurate velocity and chord-mobility analytical model, the mathematical simplicity of which represents a significant advantage because it provides necessary values by a rapid calculation process without forgoing accuracy. The model can be easily implemented in compact numerical simulations of electronic devices and associated circuits where a fast recovery of the velocity and mobility values corresponding to the local electric field and doping concentration is needed.
\end{abstract}

Keywords: InGaAs, mobility model, charge carrier mobility, drift-diffusion, semiconductor, Monte Carlo methods, transport, carrier velocity

PACS: 72.20.Fr, 72.80.Ey

\section{Introduction}

Nowadays simulators play a crucial role in the design and optimization of the performances of complex devices. The degree of precision of simulated results and the required time as well as computational resources have to be traded off in many practical cases where the complexity imposes to look for an agreement between precision and computational cost. In this search, sometimes it is useful to transform the results given by a microscopic simulator into an analytical model which facilitates their employment in the following applications. Indeed, some robust commercial packages of software support different mobility models [1],2].

A macroscopic mobility model is an average over the charge carrier velocity. In particular, in a semiconductor, electrons and holes are accelerated by electric fields, but also change the momentum as a result of several scattering processes. These scattering mechanisms typically involve lattice vibrations, impurity ions, other carriers and material imperfections. The task which brings together all those microscopic events into a macroscopic mobility model demands a kinetic approach with a rather heavy computational burden. In this way a validated [3] Monte Carlo simulator (MC) was used to calculate a set of data of the charge carrier velocity and chord-mobility for $\operatorname{In}_{0.53} \mathrm{Ga}_{0.47}$ As. In general, mobilities are functions of the electric field, lattice temperature and doping concentration, but since most devices operate at room temperature the data considered here are exclusively functions of the electric field and doping concentration. 
This set of data has been used to build an accurate analytical mobility model for $\operatorname{In}_{0.53} \mathrm{Ga}_{0.47}$ As at room temperature.

Some other analytical models [4, 5], like this one, have already shown their benefit in the modelling of carrier transport in established technologies as metal insulator semiconductor field effect transistors as well as in incipient technologies as photoconductive antennas [6-9]. Therefore the analytical model presented in this manuscript could also be used in future transport modelling of charge carriers involving $\operatorname{In}_{0.53} \mathrm{Ga}_{0.47}$ As. Certainly, a relatively high speed of carriers makes this material suitable for many applications [10-13] and an analytical mobility model might ease their study by taking advantage of compact simulations.

The current work approaches the modelling of the $\operatorname{In}_{0.53} \mathrm{Ga}_{0.47}$ As mobility by means of an analytical model presented in four sections. In Section 2 the simulator and its features are introduced. In Section 3 we present and explain the analytical model and its main characteristics. This analytical model is divided in two parts: in Subsection 3.1 we present the model for electrons while Subsection 3.2 is dedicated to the model for holes. Both models are validated with simulated results in Section 4. Finally, in Section 5 we summarize the improvements and potential applications behind the proposed analytical mobility model.

\section{The simulator}

We have simulated the transport properties of $\mathrm{In}_{0.53} \mathrm{Ga}_{0.47} \mathrm{As}$ at room temperature by adopting the standard MC simulation [14, 15] where the electronic properties of the bulk material are tied up to the scattering mechanisms and the band structure. Some interpolation was needed in gathering the values of the corresponding binary materials [16-20]. The conduction band has been considered with three non-parabolic spherical valleys (one $\Gamma$, four equivalent $\mathrm{L}$ and three equivalent $\mathrm{X}$ ). The valence band includes the non-parabolic spherical heavy-hole and light-hole bands. The scattering mechanisms that are taken into consideration in the physical model are the following ones:

- Collisions with ionized impurities (BrooksHerring model) [21].

- Transitions due to absorption and emission of polar and non-polar optical phonons.
- Collisions with acoustic elastic phonon intervalley/interband and alloy scatterings [15].

- The impact ionization has been treated in the framework of the Keldysh approach [15, 16, $22-25$.

The input parameters of the MC simulations have been adjusted to fit the experimental data of Pearsall [26]. Our MC results are also in good agreement with the simulations of Bude and Hess [3, 27]. More details about the MC model can be found in $[3]$ and references therein.

\section{The analytical model}

Due to the different behaviour exhibited by the two kinds of carriers, electrons and holes, two models have been developed. They provide the velocity and the chord-mobility as a function of the electric field using a set of parameters that depend on the concentrations of donors $N_{D}$ or acceptors $N_{\mathrm{A}}$. These quantities can be introduced in macroscopic simulators to obtain the values of the local velocity or mobility as functions of the local electric field.

\subsection{Model for electrons}

The mobility model for electrons in $\mathrm{In}_{0.53} \mathrm{Ga}_{0.47} \mathrm{As}$ has been formulated by fitting the average (drift) velocity of electrons. Equation (1) accounts for the drift velocity versus electric field. Hence Eq. (1) leads to the mobility model specified in Eq. (2). The parameters entering both equations are determined by the doping concentration $N_{D}$ and their expressions can be found in Appendix A.

1) Velocity model for electrons. Since the shape of the velocity-field curve exhibits two different slopes, we have employed the piecewise function:

$$
v_{\mathrm{e}}(|\vec{E}|)=\left\{\begin{array}{l}
\frac{A_{\mathrm{e}}\left(\sin \left(\frac{\pi|\vec{E}|}{10}\right)\right)^{b_{\mathrm{e}}}}{\mathrm{e}^{\left(c_{\mathrm{e}}|\vec{E}|^{2}\right)}} \text { if }|\vec{E}| \leq E_{\mathrm{c}, \mathrm{e}} \\
\frac{D_{\mathrm{e}}|\vec{E}|}{\left(1+\frac{|\vec{E}|-E_{\mathrm{c}, \mathrm{e}}}{3}\right)^{f_{\mathrm{e}}}} \text { if }|\vec{E}|>E_{\mathrm{c}, \mathrm{e}} .
\end{array}\right.
$$

The inflection point where the sign of the curvature changes has different threshold field $E_{c, e}$ 
depending on the value of the doping level. Another point that we would like to stress is the value of the parameter $b_{\mathrm{e}}$ : when the semiconductor is not degenerate or, in other words, $N_{\mathrm{D}}<10^{18} \mathrm{~cm}^{-3}$, the value of $b_{\mathrm{e}}$ is equal to one. Then the model contains five parameters.

2) Chord-mobility model for electrons. By dividing the drift velocity by the modulus of electric field we obtain the chord-mobility according to Eq. (2):

$$
\mu_{\mathrm{n}}(|\vec{E}|)=\frac{v_{\mathrm{e}}(|\vec{E}|)}{|\vec{E}|}
$$

The mobility model portrayed by Eq. (2) when the electric field reaches the zero value, the so-called ohmic mobility, is equal to $\mu_{\mathrm{n}}=\frac{\pi}{|\vec{E}|} A_{\mathrm{e}}$. This mobility is constant up to electric fields of about $100 \mathrm{~V} / \mathrm{cm}$, and in some cases at higher doping levels even until $1000 \mathrm{~V} / \mathrm{cm}$.

\subsection{Model for holes}

In the same manner as with the electrons, the model for holes starts with the analysis of the drift velocity leading to the mobility model for holes. Equation (3) formalizes the velocity versus the electric field for holes. Consequently Eq. (3) conducts to the mobility model detailed in Eq. (4). The parameters appearing in both equations are determined by the doping concentration of acceptors $N_{\mathrm{A}}$ and their expressions are given in Appendix B.

1) Velocity model for holes. We also define the hole velocity in $\operatorname{In}_{0.53} \mathrm{Ga}_{0.47} \mathrm{As}$ as the piecewise function:

$$
v_{\mathrm{h}}(|\vec{E}|)=\left\{\begin{array}{l}
\frac{A_{\mathrm{h}} \ln (|\vec{E}|+1)}{(|\vec{E}|+1)^{b_{\mathrm{h}}}} \text { if }|\vec{E}| \leq E_{\mathrm{c}, \mathrm{h}}, \\
\frac{C_{\mathrm{h}} \tanh (|\vec{E}|)}{\left(\ln \left(|\vec{E}|+\mathrm{d}_{\mathrm{h}}\right)\right)^{f_{\mathrm{h}}}} \text { if }|\vec{E}|>E_{\mathrm{c}, \mathrm{h}} .
\end{array}\right.
$$

The hole velocity in $\mathrm{In}_{0.53} \mathrm{Ga}_{0.47}$ As exhibits a positive slope in the whole electric field domain but its growth rate undergoes a change located at a particular value of the electric field which we call $E_{c, h}$. This parameter $E_{c, h}$ is dependent on the concentration of acceptors $N_{\mathrm{A}}$.

2) Chord-mobility model for holes. Similarly to the case for electrons, we may set down that the hole mobility is equal to $\mu_{\mathrm{p}}=A_{\mathrm{h}}$ when the electric field tends to the zero value. This ohmic mobility is practically constant till approximately $100 \mathrm{~V} / \mathrm{cm}$, and in some cases at higher doping levels even until $1000 \mathrm{~V} / \mathrm{cm}$ :

$$
\mu_{\mathrm{p}}(|\vec{E}|)=\frac{v_{\mathrm{h}}(|\vec{E}|)}{|\vec{E}|} .
$$

\section{Comparison of analytical and simulated results}

The MC simulated data have been compared with the experimental and theoretical data so as to confirm their accuracy and reliability [3, $28-39$. The results obtained for the average carrier velocities are reported in Figs. 11(a) and 2(a) for electrons and holes, respectively. Figures 1 (b) and 2(b) illustrate two particular comparisons between the simulated and the analytical velocities as well as mobilities: the first one for electrons when $N_{\mathrm{D}}=10^{16} \mathrm{~cm}^{-3}$ and the second one for holes in the case when $N_{\mathrm{A}}=10^{16} \mathrm{~cm}^{-3}$. Finally, Figs. 1(c) and 2(c) show the chord-mobility as functions of the electric field and doping concentration for electrons and holes, respectively. As expected, for low electric fields in both cases the drift velocity increases linearly. As the electric field increases, nonparabolocity effects as well as transfers to higher valleys in the case of electrons lead to a non-linear behaviour of the drift velocity. In the nonequilibrium (hot carriers) regime phonons are not able to thermalize carriers to the lattice temperature; however, they limit their velocity. When the electrons are transferred from lower to higher valleys, their average effective mass increases and their drift velocity starts to decrease.

Some interesting analytical mobility models have been proposed already [40-42]; however, they are less accurate or more complex than the one presented here. The attempt to model the velocity of electrons with an analytic expression associated with Eqs. (3) and (4) in [3] does not provide a sufficiently accurate fit. Indeed, the two branches of the piecewise function defined in [3] are not continuous at the common point represented by the threshold field $E_{\mathrm{p}}$. Additionally, this analytical model did not take into account the curvature changes versus the doping concentrations and was mainly focused in the single case $N_{\mathrm{D}}=10^{16} \mathrm{~cm}^{-3}$. 


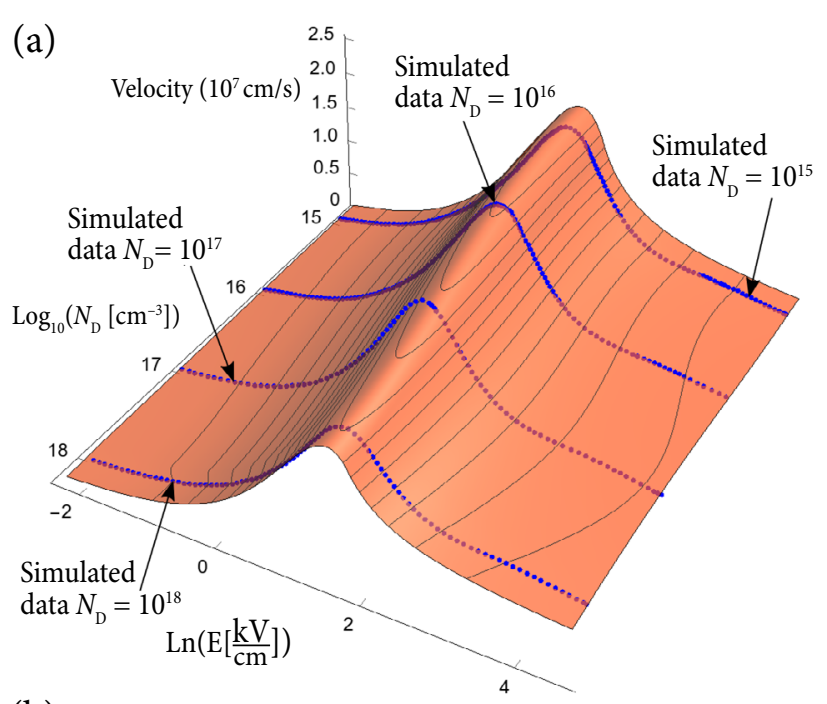

(b)

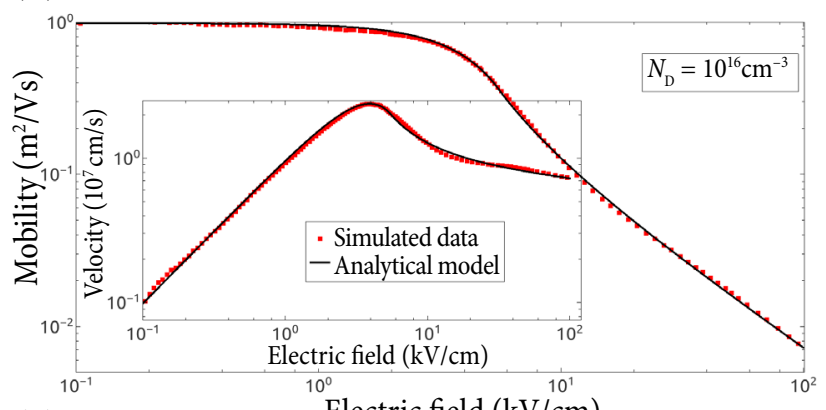

(c)

Electric field $\left(\mathrm{kV} / \mathrm{cm}^{10^{1}}\right)$

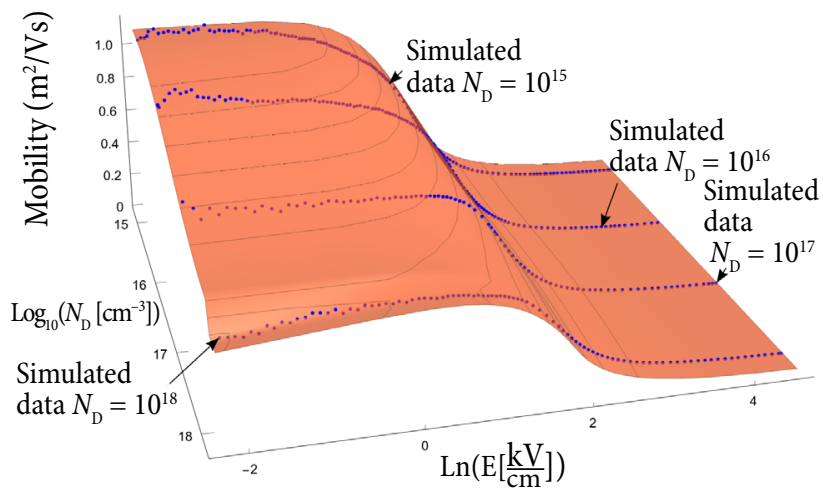

Fig. 1. (a) Analytical velocity model for electrons as a function of the electric field and donor concentration at room temperature. Some simulated profiles have been portrayed jointly. (b) Analytical model of the velocity and chord-mobility of electrons compared with the simulated data for the particular donor concentration $N_{\mathrm{D}}=10^{16} \mathrm{~cm}^{-3}$. (c) Analytical chord-mobility model for electrons as a function of the electric field and donor concentration together with some simulated data.

Moreover, the model presented in [3] was developed only for the case of electrons. Therefore, a new model able to achieve an accurate agreement with simulated data at room temperature for (a)
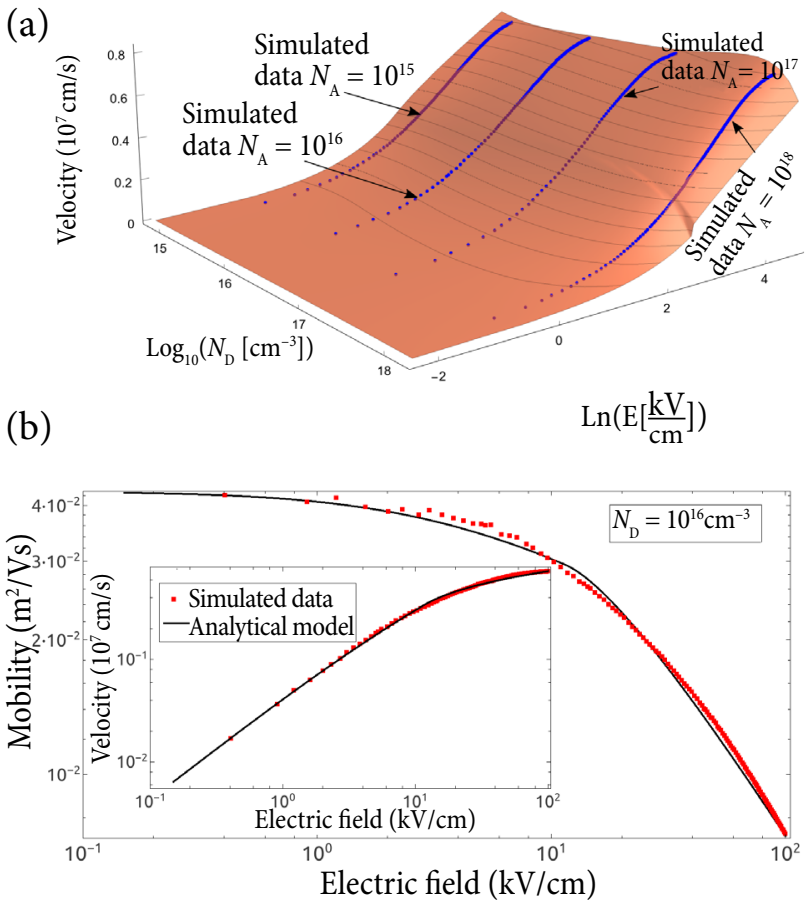

(c)

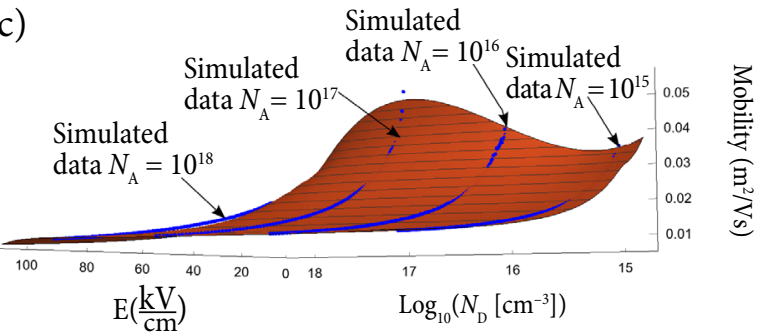

Fig. 2. Analytical velocity model for holes as a function of the electric field and acceptor concentration at room temperature. Some simulated profiles have been portrayed jointly. (b) Analytical model of velocity and chord-mobility for holes compared with the simulated data for the particular acceptor concentration $N_{\mathrm{A}}=10^{16} \mathrm{~cm}^{-3}$. (c) Analytical chord-mobility model for holes as a function of the electric field and acceptor concentration together with some simulated data.

all values of electric field and doping concentrations was necessary not only for electrons but for holes as well. Figures 1 and 2 demonstrate a very good agreement between the simulated data and the proposed analytical models for electrons and holes. Both models are equally compliant with the simulated data and they have got roughly the same computational burdens. Finally, we would like to emphasize that these computational burdens are negligible in a simulation where the mobilities need to be calculated as a function of the local electric field at every time step. 


\section{Conclusions}

An analytical mobility model of the ternary compound $\mathrm{In}_{0.53} \mathrm{Ga}_{0.47}$ As has been presented. The model concerns both kinds of carriers, i.e. electrons and holes. Monte Carlo simulation of the bulk materials under stationary and homogeneous conditions has been used as a reference in the building and validation of the model. On the one hand, the analytical model provides an accurate fit of the simulated data in a wide range of values of the electric field and doping concentrations. On the other hand, its simplicity and low computational cost make it suitable for intensive compact simulations where a macroscopic model for the charge carrier dynamics becomes mandatory.

Last, but not least, other ternary compounds as well as binary compounds could be modelled with some minor modifications using an analytical model similar to that introduced in this article.

\section{Appendix A}

\section{Parameter functions for electrons}

Parameter functions needed in the analytical models for electrons given by Eqs. (1) and (2):

$$
\begin{aligned}
& A_{\mathrm{e}}\left(N_{\mathrm{D}}\right)=0.115\left(\log _{10}\left(N_{\mathrm{D}}\right)\right)^{3}-5.7\left(\log _{10}\left(N_{\mathrm{D}}\right)\right)^{2} \\
& -503.35+93.415 \log _{10}\left(N_{\mathrm{D}}\right), \\
& b_{\mathrm{e}}\left(N_{\mathrm{D}},|\vec{E}|\right)=\left\{\begin{array}{l}
1 \quad \text { if } N_{\mathrm{D}}<10^{18} \mathrm{~cm}^{-3}, \\
1+\frac{2\left(1-\mathrm{e}^{-100|\vec{E}|}\right)}{25} \text { if } N_{\mathrm{D}} \geq 10^{18} \mathrm{~cm}^{-3}
\end{array}\right. \\
& c_{\mathrm{e}}\left(N_{\mathrm{D}}\right)=-55.59552+36.71459 \sqrt{\log _{10}\left(N_{\mathrm{D}}\right)} \\
& -6.80779 \log _{10}\left(N_{\mathrm{D}}\right)+0.06905\left(\log _{10}\left(N_{\mathrm{D}}\right)\right)^{2}, \\
& D_{\mathrm{e}}\left(N_{\mathrm{D}},|\vec{E}|\right)=\frac{A_{\mathrm{e}}\left(N_{\mathrm{D}}\right)\left[\sin \left(\frac{\pi E_{\mathrm{c}, \mathrm{e}}\left(N_{\mathrm{D}}\right)}{10}\right)\right]_{\rfloor}^{b_{\mathrm{e}}\left(N_{\mathrm{D}}, \vec{E} \mid\right)}}{E_{\mathrm{c}, \mathrm{e}}\left(N_{\mathrm{D}}\right) \mathrm{e}^{\left(c_{\mathrm{e}}\left(N_{\mathrm{D}}\right) E_{\mathrm{c}, \mathrm{e}}^{2}\left(N_{D}\right)\right)},} \\
& E_{\mathrm{c}, \mathrm{e}}\left(N_{\mathrm{D}}\right)=0,166667 \log _{10}\left(N_{\mathrm{D}}\right)+3, \\
& f_{\mathrm{e}}\left(N_{\mathrm{D}}\right)=0,005\left(\log _{10}\left(N_{\mathrm{D}}\right)\right)^{2}-0.185 \log _{10}\left(N_{\mathrm{D}}\right) \\
& +2.780 .
\end{aligned}
$$

\section{Appendix B}

Parameter functions for holes

Parameter functions needed in the analytical models for holes given by Eqs. (3) and (4):

$$
\begin{aligned}
& A_{\mathrm{h}}\left(N_{\mathrm{A}}\right)=0.3295\left(\log _{10}\left(N_{\mathrm{A}}\right)\right)^{2}-0.006833\left(\log _{10}\left(N_{\mathrm{A}}\right)\right)^{3} \\
& +28.155-5.279667 \log _{10}\left(N_{\mathrm{A}}\right) \\
& b_{\mathrm{h}}\left(N_{\mathrm{A}}\right)=134.75-25.51667 \log _{10}\left(N_{\mathrm{A}}\right) \\
& +1.6\left(\log _{10}\left(N_{\mathrm{A}}\right)\right)^{2}-0.033333\left(\log _{10}\left(N_{\mathrm{A}}\right)\right)^{3} \\
& C_{\mathrm{h}}\left(N_{\mathrm{A}}\right)=96.14446-0.023396\left(\log _{10}\left(N_{\mathrm{A}}\right)\right)^{3} \\
& +1.12725\left(\log _{10}\left(N_{\mathrm{A}}\right)\right)^{2}-18.04413 \log _{10}\left(N_{\mathrm{A}}\right) \\
& d_{\mathrm{h}}\left(N_{\mathrm{A}}\right)=3.5-0.5 \log _{10}\left(N_{\mathrm{A}}\right), \\
& E_{\mathrm{c}, \mathrm{h}}\left(N_{\mathrm{A}}\right)=\log _{10}\left(N_{\mathrm{A}}\right)-5, \\
& f_{\mathrm{h}}\left(N_{\mathrm{A}}\right)=287.18-54.538333 \log ^{10}\left(N_{\mathrm{A}}\right) \\
& +3.43\left(\log _{10}\left(N_{\mathrm{A}}\right)\right)^{2}-0.071667\left(\log _{10}\left(N_{\mathrm{A}}\right)\right)^{3} .
\end{aligned}
$$

\section{Acknowledgements}

The authors would like to thank T. Gonzalez and J. Mateos from the University of Salamanca (Spain) for the development of the Monte Carlo code, and G. Sabatini from the University of Montpellier (France) for the simulations of the bulk input data. The first author would like to thank Galya Klochok for her patience, understanding and cooperation which have allowed this publication.

\section{References}

[1] Atlas, https://www.silvaco.com

[2] Comsol Multiphysics, https://www.comsol.com/

[3] S. Karishy, P. Ziadé, G. Sabatini, H. Marinchio, C. Palermo, L. Varani, J. Mateos, and T. Gonzalez, Review of electron transport properties in bulk InGaAs and InAs at room temperature, Lith. J. Phys. 55(4), 305-314 (2015).

[4] R. Caughey and D.M. Thomas, Carrier mobilities in silicon empirically related to doping and field, IEEE Proc. 55(12), 2192-2193 (1967). 
[5] C. Lombardi, S. Manzini, A. Saporito, and M. Vanzi, A physically based mobility model for numerical simulation of nonplanar devices, IEEE Trans. Comput. Aided Design Integr. Circuits Syst. 7(11), 1164-1171 (1988).

[6] E. Moreno, M. Pantoja, F. Ruiz, J. Roldń, and S. García, On the numerical modeling of terahertz photoconductive antennas, J. Infrared Millim. Terahertz Waves 35(5), 432-444 (2014).

[7] E. Moreno, M. Pantoja, S. Garcia, A. Bretones, and R. Martin, Time-domain numerical modeling of $\mathrm{THz}$ photoconductive antennas, IEEE Trans. Terahertz Sci. Technol. 4(4), 490-500 (2014).

[8] E. Moreno, Z.Hemmat, J.B. Roldán, M.F. Pantoja, A.R. Bretones, and S.G. García, Time-domain numerical modeling of terahertz receivers based on photoconductive antennas, J. Opt. Soc. Am. B 32(10), 2034-2041 (2015).

[9] E. Moreno, Z. Hemmat, J.B. Roldán, M.F. Pantoja, A.R. Bretones, S.G. García, and R. Faez, Implementation of open boundary problems in photo-conductive antennas by using convolutional perfectly matched layers, IEEE Trans. Antennas Propag. 64(11), 4919-4922 (2016).

[10]T. Ishibashi, Y. Muramoto, T. Yoshimatsu, and H. Ito, Uni-traveling-carrier photodiodes for terahertz applications, IEEE J. Sel. Top. Quantum Electron. 20(6), 79-88 (2014).

[11]Q. Chen, Y. Huang, X. Duan, F. Liu, C. Kang, Q. Wang, J. Wang, X. Zhang, and X. Ren, Highspeed uni-traveling-carrier photodetector with the new design of absorber and collector, in: Proceedings of the 2015 Opto-Electronics and Communications Conference (OECC) (2015) pp. 1-3.

[12]F. Liu, Y. Huang, C. Kang, Q. Chen, X. Duan, and $\mathrm{X}$. Ren, High speed and high responsivity dual-absorption InGaAs/InP UTC-PDs, in: Proceedings of the 2015 Opto-Electronics and Communications Conference (OECC) (2015) pp. 1-3.

[13]X. Zhao, C. Heidelberger, E.A. Fitzgerald, and J.A. del Alamo, Source/drain asymmetry in InGaAs vertical nanowire MOSFETs, IEEE Trans. Electron Dev. 64(5), 2161-2165 (2017).
[14]I.M. Sobol, The Monte Carlo Method, Little Mathematics Library (Mir Publishers, Moscow, 1975).

[15]C. Jacoboni and L. Reggiani, The Monte Carlo method for the solution of charge transport in semiconductors with applications to covalent materials, Rev. Mod. Phys. 55, 645-705 (1983).

[16]M.V. Fischetti, Monte Carlo simulation of transport in technologically significant semiconductors of the diamond and zinc-blende structures. I. Homogeneous transport, IEEE Trans. Electron Dev. 38(3), 634-649 (1991).

[17]J. Mateos, T. Gonzalez, D. Pardo, V. Hoel, H. Happy, and A. Cappy, Improved Monte Carlo algorithm for the simulation of $\delta$-doped AlInAs/GaInAs HEMTs, IEEE Trans. Electron Dev. 47(1), 250-253 (2000).

[18]K.F. Brennan and D.H. Park, Theoretical comparison of electron real space transfer in classical and quantum two-dimensional heterostructure systems, J. Appl. Phys. 65(3), 1156-1163 (1989).

[19]S. Adachi, Physical Properties of III-V Semiconductor Compounds: InP, InAs, GaAs, GaP, In $G a A s$, and InGaAsP (Wiley Online Library, 2005).

[20]O. Madelung, Semiconductors: Data Handbook (Springer, Berlin, 2003).

[21]K. Aoki, Nonlinear Dynamics and Chaos in Semiconductors (CRC Press, 2000).

[22] G.M. Dunn, G.J. Rees, J.P.R. David, S.A. Plimmer, and D.C. Herbert, Monte Carlo simulation of impact ionization and current multiplication in short GaAs $\mathrm{p}^{+} \mathrm{in}^{+}$diodes, Semicond. Sci. Technol. 12(1), 111 (1997).

[23] G.M. Dunn, A. Phillips, and P.J. Topham, Current instability in power HEMTs, Semicond. Sci. Technol. 16(7), 562 (2001).

[24]B.G. Vasallo, J. Mateos, D. Pardo, and T. González, Influence of trapping-detrapping processes on shot noise in nondegenerate quasi-ballistic transport, Semicond. Sci. Technol. 17(5), 440 (2002).

[25] K. Kalna and A. Asenov, Gate tunnelling and impact ionisation in sub $100 \mathrm{~nm}$ PHEMTs, IEICE Trans. Electron. E86-C(3), 330-335 (2003). 
[26]T.P. Pearsall, Impact ionization rates for electrons and holes in $\mathrm{Ga}_{0.47} \mathrm{In}_{0.53}$ As, Appl. Phys. Lett. 36(3), 218-220 (1980).

[27]J. Bude and K. Hess, Thresholds of impact ionization in semiconductors, J. Appl. Phys. 72(8), 3554-3561 (1992).

[28]T.H. Windhorn, L.W. Cook, and G.E. Stillman, The electron velocity field characteristic for $\mathrm{n}$ - $\mathrm{In}_{0.53} \mathrm{Ga}_{0.47}$ As at $300 \mathrm{~K}$, IEEE Electron Dev. Lett. 3(1), 18-20 (1982).

[29]J.L. Thobel, L. Baudry, A. Cappy, P. Bourel, and R. Fauquembergue, Electron transport properties of strained $\mathrm{In}_{x} \mathrm{Ga}_{1-x}$ As, Appl. Phys. Lett. 56(4), 346-348 (1990).

[30]Y. Hori, Y. Ando, Y. Miyamoto, and O. Sugino, Effect of strain on band structure and electron transport in InAs, Solid State Electron. 43(9), 1813-1816 (1999).

[31]J.H. Marsh, Effects of compositional clustering on electron transport in $\mathrm{In}_{0.53} \mathrm{Ga}_{0.47}$ As, Appl. Phys. Lett. 41(8), 732-734 (1982).

[32]M.A. Haase, V.M. Robbins, N. Tabatabaie, and G.E. Stillman, Subthreshold electron velocityfield characteristics of $\mathrm{GaAs}$ and $\mathrm{In}_{0.53} \mathrm{Ga}_{0.47} \mathrm{As}$, J. Appl. Phys. 57(6), 2295-2298 (1985).

[33]M. Littlejohn, K. Kim, and H. Tian, in: Properties of Lattice-Matched and Strained Indium Gallium Arsenide, ed. P. Bhattacharya (INSPEC, London, U. K., 1993).

[34]W.K. Ng, C.H. Tan, J.P.R. David, P.A. Houston, M. Yee, and J.S. Ng, Temperature dependent low-field electron multiplication in $\mathrm{In}_{0.53} \mathrm{Ga}_{0.47} \mathrm{As}$, Appl. Phys. Lett. 83(14), 2820-2822 (2003).

[35]C.H. Tan, G.J. Rees, P.A. Houston, J.S. Ng, W.K. Ng, and J.P.R. David, Temperature depend- ence of electron impact ionization in $\operatorname{In}_{0.53} \mathrm{Ga}_{0.47} \mathrm{As}$, Appl. Phys. Lett., 84(13), 2322-2324 (2004).

[36] G. Satyanadh, R.P. Joshi, N. Abedin, and U. Singh, Monte Carlo calculation of electron drift characteristics and avalanche noise in bulk InAs, J. Appl. Phys. 91(3), 1331-1338 (2002).

[37]M. Isler, Phonon-assisted impact ionization of electrons in $\operatorname{In}_{0.53} \mathrm{Ga}_{0.47} \mathrm{As}$, Phys. Rev. B 63, 115209 (2001).

[38]V. Balynas, A. Krotkus, A. Stalnionis, A.T. Gorelionok, N.M. Shmidt, and J.A. Tellefsen, Timeresolved, hot-electron conductivity measurement using an electro-optic sampling technique, Appl. Phys. A 51(4), 357-360 (1990).

[39] L. Amer, C. Sayah, B. Bouazza, A. Guen-Bouazza, N. Chabane-Sari, and C. Gontrand, Analyse du phénomène de transport électronique dans l'InAs et le GaAs par la méthode de Monte Carlo pour la conception d'un transistor PHEMT, in: CISTEMA'2003 (Université de Tlemcen).

[40]J. Jogi, S. Sen, M. Gupta, and R. Gupta, An analytical 2D model for drain-induced barrier lowering in subquarter micrometer gate length InAlAs/ InGaAs/InAlAs/InP LMHEMT, Microelectronics J. 33(8), 633-638 (2002).

[41]Y. Huang, J. Xu, L. Wang, and S. Zhu, A physical model on electron mobility in InGaAs nMOSFETs with stacked gate dielectric, Microelectron. Reliab. 55(2), 342-346 (2015).

[42]G.B. Beneventi, S. Reggiani, A. Gnudi, E. Gnani, A. Alian, N. Collaert, A. Mocuta, A. Thean, and G. Baccarani, A TCAD low-field electron mobility model for thin-body InGaAs on InP MOSFETs calibrated on experimental characteristics, IEEE Trans. Electron Dev. 62(11), 3645-3652 (2015).

\title{
TIKSLUS ANALIZINIS NEPUSIAUSVYRINIO DREIFO GREIČIO IR CHORDINIO JUDRIO In ${ }_{0,53} \mathrm{Ga}_{0,47}$ As MODELIS
}

\author{
E. Moreno ${ }^{\text {a }}$, L. Varani ${ }^{\text {b }}$ \\ ${ }^{a}$ Čilés universitetas, Santjagas, Čile \\ ${ }^{\mathrm{b}}$ Monpeljè universitetas, Monpeljè, Prancüzija
}

